EDITORIAL

\title{
Chiasmus as an Alternative Pattern of Teaching
}

\section{Dhouha Bacha', Wael Ferjaoui2* (D), Lasaad Gharbi', Sana Ben Slama' and Ahlem Lahmar}

${ }^{1}$ Department of Pathology, Mongi Slim University Hospital, Faculty of Medicine of Tunis, University of Tunis El Manar, Tunisia

${ }^{2}$ Department of General Surgery, Mongi Slim University Hospital, Faculty of Medicine of Tunis, University of Tunis El Manar, Tunisia

*Corresponding author: Wael Ferjaoui, Department of General Surgery, Mongi Slim University Hospital, Faculty of Medicine of Tunis, University of Tunis El Manar, Tunisia, Tel: +216-524-300-99

Etymologically the word chiasm comes from the Greek word khiasmós which means crossing.

The chiasm construction is a repetition crossing 2 segments $A-B$ around a center $C$ creating an $A B / C / B A$ structure.

This is a rhetorical figure of speech expressing repetition.

In linguistics, the purpose of repetition is the emphasis on the message to be conveyed.

In pedagogy, the 3 goals of repetition are: Consolidation, deepening and feedback.

In the domain of procedural knowledge, repetition makes it possible to create automatisms.

Chiasmus is already reported in teaching [1].

We propose a new form of chiasmus as follows: Mini method 1 (15 to $20 \mathrm{~min}$.) -Mini case 1 (15 min) -pause (5 $\mathrm{min}$ ) -Mini case 2 (15 min) Mini method 2 (15-20 min) -

The prefix Mini here signifies a contraction in time.

We call Mini method: The methods without a case study, they can be: Mini Lecture, Mini talk chalk or Mini learning by keywords.

We call Mini case: The learning around a clinical case, it can be: Mini Challenge-based learning (CBL), Mini teaching by clinical case guide, Mini bedside case.

In order to promote deep learning, the Mini case 2 must be more complex than its counterpart 1 and the Mini method 2 more in-depth than its counterpart 1.
In practice, it can be: Mini lecture-Mini CBL-pause-Mini CBL-Mini talk chalk.

The Mini lecture-Mini CBL association can be benefic. Indeed, multiple interactive mini-cases with an abbreviated lecture improved immediate mastery of learning objectives compared to a traditional lecture format [2].

Elsewhere it may be Mini talk chalk-mini bedside case-pause-Mini CBL-Mini lecture.

The association Mini talk chalk and Mini bedside case maximizes the opportunities for clinical learning [3].

Our chiasm has more repetition than Mini lecture-Mini CBL (ref) or Mini talk chalk-mini bedside case.

The chiasm rhythm makes it possible to immediately bounce back on the activities of the break to discuss the Mini case 2. This model must respond to a prior scripting.

Our model meets the educational requirement of: contextualization-decontextualization-recontextualization.

We have described elsewhere the educational requirements of the Mini methods and the advantage of inserting the image tool on hybrid media in order to create: action-retro action-inter action [4].

Our model is anchored in old methods (bedside) while remaining open to modern methods (possibility of inserting cases in simulation).

\footnotetext{
Citation: Bacha D, Ferjaoui W, Gharbi L, Slama SB, Lahmar A (2020) Chiasmus as an Alternative Pattern of Teaching. Int J Cogn Behav 3:010. doi.org/10.23937/2690-3172/1710010 in any medium, provided the original author and source are credited.
} 


\section{Acknowledgements}

None.

\section{Conflict of Interest}

None.

\section{References}

1. [AMA] MED EDU Innovation Challange | Medical Education | Medical School [Internet]. Scribd. Disponible sur.
2. Marshall L, Nykamp D, Momary K (2014) Impact of abbreviated lecture with interactive mini-cases vs traditional lecture on student performance in the large classroom. Am J Pharm Educ 78: 189.

3. Pitt M, Orlander J (2017) Bringing mini-chalk talks to the bedside to enhance clinical teaching. Med Educ Online 22: 1264120.

4. Dhouha Bacha, Omrani S, Saadia B, Gharbi L, Talbi G, et al. (2020) The Mini Method as an Alternative Approach in Medical Education - A comprehensive Review of the Literature. Open Access Journal of Oncology and Medicine 3: 292-294. 